\title{
Mirnome analysis reveals novel molecular determinants in the pathogenesis of diet-induced nonalcoholic fatty liver disease
}

\author{
Anna Alisi ${ }^{1, *}$, Letizia Da Sacco ${ }^{2, *}$, Giovannella Bruscalupi $^{3}$, Fiorella Piemonte ${ }^{4}$, Nadia Panera ${ }^{1}$, Rita De Vito ${ }^{5}$, \\ Silvia Leoni ${ }^{3}$, Gian Franco Bottazzo ${ }^{6}$, Andrea Masotti ${ }^{2, \dagger}$ and Valerio Nobili ${ }^{1, \dagger}$
}

Nonalcoholic fatty liver disease (NAFLD) is an emerging disease with a broad spectrum of liver conditions. The complex molecular pathogenesis of NAFLD is still unclear. In this study, we conducted an analysis of microRNA (miRNA) expression profiles in liver of rats made NAFLD by different diets. To this aim, Sprague-Dawley rats were fed ad libitum for 3 months with different diets: standard diet (SD), diet enriched in fats and low in carbohydrates (HFD), SD with high fructose (SD-HF) and diet with high levels of fats and fructose (HFD-HF). Our results demonstrated that the treatment with different dietetic regimens caused a significant increase of the body weight and the alteration of some metabolic parameters compared with control animals, as well as various liver injuries. The miRNAs analysis showed the significant downregulation of three miRNAs (miR-122, miR-451 and miR-27) and the upregulation of miR-200a, miR-200b and miR-429 in HFD, SD-HF and HFD-HF rats. Besides, miR-21 expression was significantly decreased only in fructose-enriched diets. These miRNAs target molecules involved in the control of lipid and carbohydrate metabolism, signal transduction, cytokine and chemokine-mediated signaling pathway and apoptosis. Western blot analysis of PKC $\delta$, LITAF, ALDOLASE-A, P38MAPK, PTEN, LIPIN1, EPHRIN-A1, EPHA2 and FLT1 showed a diet-induced deregulation of all these proteins. Interestingly, the expression pattern of LITAF, PTEN, LIPIN1, EPHRIN-A1, EPHA2 and FLT1 might be well explained by the trend of their specific mRNAs, by potentially regulatory miRNAs, or both. In conclusion, we highlight for the first time the potential involvement of novel determinants (miRNAs and proteins) in the molecular pathogenesis of diet-induced NAFLD. Laboratory Investigation (2011) 91, 283-293; doi:10.1038/labinvest.2010.166; published online 18 October 2010

KEYWORDS: microRNA; NAFLD; steatohepatitis; targets

Nonalcoholic fatty liver disease (NAFLD) is the most common broad-spectrum liver disease in the United States, and it is quickly becoming a worldwide health problem. ${ }^{1}$ NAFLD ranges from fatty liver to nonalcoholic steatohepatitis (NASH), that often precedes liver fibrosis, cirrhosis and hepatocellular carcinoma. ${ }^{2-4}$

It is well known that the prevalence of NAFLD associates with several risk factors including obesity, metabolic syndrome and type 2 diabetes, and it is interrelated in a complex manner with insulin resistance. ${ }^{5,6}$ To date, the molecular pathogenesis of NAFLD is still unclear. However, the advent of new high-throughput technologies (ie microarrays) is enabling the deep investigation of the complex network of interactions between gene/proteins and biological response during the development of simple steatosis and progression to NASH and fibrosis. ${ }^{7-9}$ In fact, in recent years, significant information in the understanding of NAFLD pathogenesis has been gained by examining the data accumulated by high-throughput technologies. ${ }^{10,11}$ Often a single approach is not sufficient to give a comprehensive picture of hepatic dysfunctions occurring in NAFLD, and somewhat, a multiple-approach combination of different techniques is

\footnotetext{
'Unit of Metabolic and Autoimmune Liver Diseases, Bambino Gesù Children's Hospital and Research Institute, Rome, Italy; ${ }^{2}$ Unit of Gene Expression-Microarrays, Bambino Gesù Children's Hospital and Research Institute, Rome, Italy; 'Department of Biology and Biotechnology 'C. Darwin', 'La Sapienza' University, Rome, Italy; ${ }^{4}$ Unit of Molecular Medicine, Bambino Gesù Children's Hospital and Research Institute, Rome, Italy; ${ }^{5}$ Unit of Pathological Anatomy, Bambino Gesù Children's Hospital and Research Institute, Rome, Italy and 'Immunology Area, Bambino Gesù Children's Hospital and Research Institute, Rome, Italy

Correspondence: Dr A Alisi, PhD, Unit of Metabolic and Autoimmune Liver Diseases, Bambino Gesù Children's Hospital and Research Institute, S. Onofrio 4 Square, 00165 Rome, Italy.

E-mail: anna.alisi@opbg.net

*These authors contributed equally to this work.

'These authors contributed equally to this work.
} 
recommended. ${ }^{12}$ Calvert et al ${ }^{13}$ showed a good example of integration of system biology with reverse-phase protein microarray analysis to define genetic architecture of NAFLD. Very recently, also microRNA (miRNA) expression profiling has provided important insights into molecular mechanisms involved in the development of NAFLD. ${ }^{14}$

From the revolutionary discovery of the first miRNA in Caenorhabditis elegans (1993) to date, hundreds of distinct miRNAs in mammalian cells have been identified. ${ }^{15,16}$ MiRNAs are 'noncoding' RNAs that negatively regulate, at a post-transcriptional level, the synthesis of several proteins implicated in many different cellular processes, including cell proliferation, differentiation and apoptosis. ${ }^{17}$ The human genome is now predicted to encode $\sim 1000$ miRNAs that likely regulate at least one third of all human transcripts. ${ }^{18}$ Nowadays, miRNAs are emerging as important contributors to many normal homeostatic processes, including regulation of metabolic pathways, cellular stress, immune defense and inflammation. ${ }^{19-21}$ Interestingly, alterations in miRNA expression profiles have also been observed in a variety of chronic diseases, such as cancer and diabetes. ${ }^{22,23}$ Furthermore, miRNA expression patterns and their role in liver diseases are another recent acquisition. ${ }^{24,25}$ In fact, although a differential miRNA expression pattern was found in the liver of hepatitis $\mathrm{B}$ and $\mathrm{C}$ virus infected individuals, and specific miRNAs (ie miR-122) have been associated to cirrhosis and hepatocellular carcinoma, the association between miRNAs and NAFLD is still poor known. ${ }^{25}$ Four recent reports analyzed miRNA profiles in animal models and human NAFLD. ${ }^{26-29} \mathrm{Li}$ et $a l^{26}$ found that miR-34a, miR-31, miR-103, miR-107, miR-194, miR-335-5p, miR-221 and miR-200a were upregulated, whereas miR-29c, miR-451 and miR-21 were downregulated in liver of mice with diet-induced NASH. However, the expression levels of specific hepatic miRNAs during NAFLD pathogenesis are still controversial. For example, the expression of miR-122 may be either downregulated in humans and mice, or upregulated in rats. ${ }^{26-28}$ All these findings confirm a connection between definite miRNA signatures and pathogenesis of NAFLD, but also suggest that further investigations are required.

We hypothesized that miRNA profiles of liver tissues derived from a model that mimics all the spectrum of histological features of human NAFLD (ie steatosis, ballooning and fibrosis) may elucidate the molecular mechanisms involved in the development of fatty liver and its progression to NASH. We choose, as animal models of NAFLD, rats treated with different dietetic regimens, a standard diet (SD) or a high-fat diet (HFD) both enriched or not with fructose (HF). Our results demonstrated that a dysregulated miRNA expression pattern is associated with different histological damages and metabolic derangement in NAFLD. Furthermore, we identified novel disease-specific miRNAs and proteins that in a near future might be either considered as potential targets for therapeutic intervention or early predictors of NASH.

\section{MATERIALS AND METHODS}

\section{Animals and Diets}

Twenty-four Sprague-Dawley rats weighing 100-120 g each were obtained from (Harlan Italy, San Pietro al Natisone, UD, Italy). Rats were fed four different diets: a SD and a high-fat diet (HFD) both enriched or not with fructose (HF). SD was composed of $5 \%$ of energy derived from fat, $18 \%$ from proteins and $77 \%$ from carbohydrates all for a total of $3.3 \mathrm{kcal} / \mathrm{g}$, whereas HFD contained $58 \%$ of energy derived from fat, $18 \%$ from protein and $24 \%$ from carbohydrates, for a total of $5.6 \mathrm{kcal} / \mathrm{g}$. Fructose was added to the drinking water $(30 \% \mathrm{w} / \mathrm{v})$. After 3 months of treatment, rats were killed. Liver tissue and portal blood samples were collected to perform the experiments. Rats were housed in plastic cages under standard conditions with free access to water and food, and treated in agreement with the guidelines of the local committee for care and treatment of laboratory animals.

\section{Biochemical Determinations}

Serum samples were obtained from portal blood after $2000 \mathrm{~g}$ centrifugation at $4^{\circ} \mathrm{C}$ for $15 \mathrm{~min}$. Serum was used to measure levels of alanine aminotransferase (ALT), triglycerides, total cholesterol, glucose and insulin, by ELISA kits according to the manufacturer's instructions. ALT assay kit was purchased from Randox Laboratories (Antrim, UK); triglycerides and cholesterol assay kits were purchased from Cayman Chemical (Ann Arbor, MI, USA); glucose assay kit was purchased from Abcam (Cambridge, MA, USA) and rat insulin enzyme immunoassay kit from SPI-BIO (France).

\section{Liver Histology and Immunohistochemistry}

Liver was fixed in $40 \mathrm{~g} / \mathrm{l}$ formaldehyde, embedded in paraffin. A measure of $5 \mu \mathrm{m}$ sections were stained with hematoxylin and eosin (H\&E) and Van Gieson (VG) and examined by a pathologist. The presence and degree of steatosis was evaluated on H\&E-stained sections as previously described ${ }^{30}$; whereas, VG staining was used to evaluate the presence of fibrosis.

Immunohistochemistry was performed as described by the manufacturer by using antibodies specific for rabbit antiCollagen VI (Rockland, Gilbertsville, PA, USA) and mouse anti-CD163 (Dako, Carpinteria, CA, USA). Detection of the primary antibody was carried out by using the appropriate secondary biotinylated antibody (Vector Laboratories, Bridgeport, New Jersey, USA) and the peroxidase DAB kit (Dako).

\section{miRNA Microarray}

Total RNA from liver tissue of each animal was extracted with TRIzol reagent according to the manufacturer's protocol (Invitrogen, San Giuliano Milanese, Italy). A measure of $5 \mu \mathrm{g}$ of RNA were used for miRNA microarray (LC-Sciences, Houston, TX, USA). In particular, at least three samples of each group were labeled with $\mathrm{Cy} 3$ and Cy5 fluorescent dyes and hybridized to a single array ( $\mu$ ParaFlo microfluidics chip) 
that included 350 mature rat miRNA probes (miRNABase 11.0), and 50 probes for quality control. After hybridization and signal detection, background was subtracted from raw data and values were normalized using the LOWESS (locally weighted regression) method. ${ }^{31}$ Significant miRNAs (analysis of variance (ANOVA), $P$-value $\leq 0.05)$, with a fold-change expression variation of at least $1.4(0.7>$ fold change $>1.4)$ in diet-treated rat with respect to control, were clusterized using average-linkage algorithm and Euclidian distance correlation as similarity measure. Hierarchical clustering was performed using GeneSpring (Ver 7.2) software.

\section{miRNA Expression by qRT-PCR}

Quantitative real-time PCR was performed to validate differentially expressed miRNAs $(P$-value $\leq 0.05)$, using stemloop primers specifically designed for the analysis of mature miRNA assays. cDNA was synthesized from $5 \mathrm{ng}$ of total RNA using TaqMan MiRNA Reverse Transcriptase (Applied Biosystems, Foster City, CA, USA) according to the manufacturer's instructions. RT-PCR was performed with the RT product, TaqMan Universal Master Mix, primers and probe mix (TaqMan MiRNA Assay kit, Applied Biosystems). Each PCR reactions were run in triplicate. Amplification and detection were performed with the ABI PRISM 7700 Sequence Detection System. The endogenous control U6 (ABI) was used for normalization and the relative quantity of miRNAs was calculated by $2^{-\Delta \Delta C T}$ method, comparing diet-treated rats to control. ${ }^{32}$

\section{Identification of Potential miRNA Targets}

Gene targets of significant differentially expressed miRNAs were identified by prediction software including MiRanda 3.0 from miRBase Release 12.0 (http://www.mirbase.org), TargetScan 5.1 (http://www.targetscan.org/) and miRecords (http://miRecords.umn.edu/miRecords/). All miRNA targets were categorized into functional classes using gene ontology hierarchy (http://www.geneontology.org and DAVID Bioinformatics Resources). Targets of each miRNA, predicted by miRecords, were investigated to share common biological processes and derived their statistical enrichment using PANTHER classification system (Celera Genomics, Foster City, CA, USA). ${ }^{33}$

\section{Western Blotting}

Liver tissue samples $(100 \mathrm{mg}$ ) were minced and lysed in icecold RIPA buffer supplemented with protease inhibitors as already described. ${ }^{34}$ Briefly, samples were electrophoresed in 10-12.5\% SDS-PAGE and transferred onto PVDF membrane (Amersham, Germany), blocked with 5\% nonfat dried milk and incubated with appropriate primary and secondary antibodies. Immunoblots were visualized with the ECL detection system (Amersham). All used primary antibodies were purchased from Santa Cruz Santa Cruz Biotech. (CA, USA). Quantification of bands was determined by densito- metric analysis, final densitometric readings were normalized against $\beta$-actin.

\section{mRNA Expression by RT-PCR}

The expression levels of nine mRNAs and the endogenous control gene ActB were measured in each sample by real-time PCR using TaqMan Gene Expression assays on ABI PRISM 7900HT Sequence Detection System (Applied Biosystems) (see Supplementary Table S1). For each sample, three replicates were run for each gene in a 96-well format plate. A measure of $1 \mu \mathrm{g}$ of total RNA from each total RNA sample was used to generate cDNA using the high-capacity cDNA Archiving Kit (Applied Biosystems). Real-time PCR reactions were carried out following the manufacturer's protocol. Gene expression values were determined as $\Delta \mathrm{Ct}\left(\mathrm{Ct}_{(\text {sample })}-\mathrm{Ct}_{(\mathrm{GAPDH})}\right)$ and relative quantities between different samples were determined as $\Delta \Delta \mathrm{Ct}$ $\left(\Delta \mathrm{Ct}_{(\text {sample1 })}-\Delta \mathrm{Ct}_{(\text {sample2 })}\right) .^{32}$

\section{Statistical Analysis}

Statistical comparison between various groups was performed by one-way ANOVA, with least significant difference post hoc test, using the SPSS software (version 12.0.2). Comparisons were made between means from three independent experiments and differences were considered significant when $P$-values were $<0.05$.

\section{RESULTS \\ Body Weight, Metabolic Variables and Liver Histology After Different Dietetic Treatments}

At the end of the 3-month diet, we observed different patterns of the body weight and metabolic alterations among the various groups (Figure 1a-f). HFD, SD-HF and HFD-HF rats gained more body weight than those consuming the SD, although no difference in body weight among the groups feeding hypercaloric diets was found. Serum levels of ALT and triglycerides were greatly influenced by high-fat diet alone or in combination with fructose, whereas levels of total cholesterol were unchanged by diets. No significant differences in serum glucose levels were found, whereas serum levels of insulin were significantly enhanced by all dietetic regimens with respect to the controls $(\mathrm{SD})$.

As revealed by the staining with $\mathrm{H} \& \mathrm{E}$ (Figure 2), liver of rats fed with different dietetic regimens showed histological changes with respect to SD livers, which have no evident abnormalities. The HFD tissues present a mild steatosis, whereas the SD-HF group was essentially characterized by ballooning in numerous hepatocytes. The combination of high-fat diet with an excess fructose intake (HFD-HF) provokes ballooning associated to mild steatosis. This analysis revealed no significant inflammation. The VG staining demonstrated that only liver tissue from HFD-HF rats showed the presence of rare fibrosis (data not shown). To better evaluate the liver damage, we performed an immunohistochemical analysis of collagen VI (marker of fibrosis) and 
a

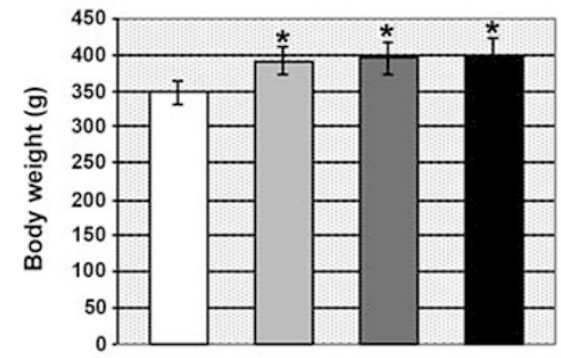

d
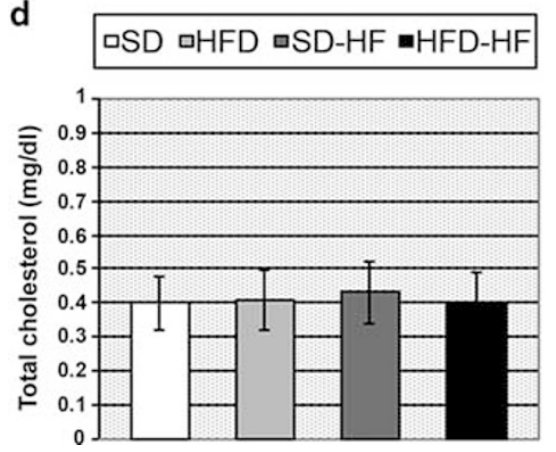

b
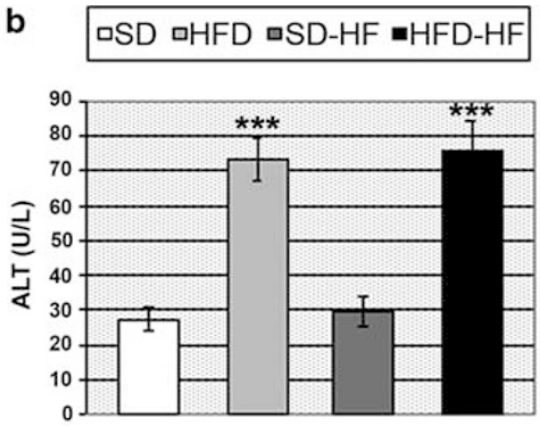

e
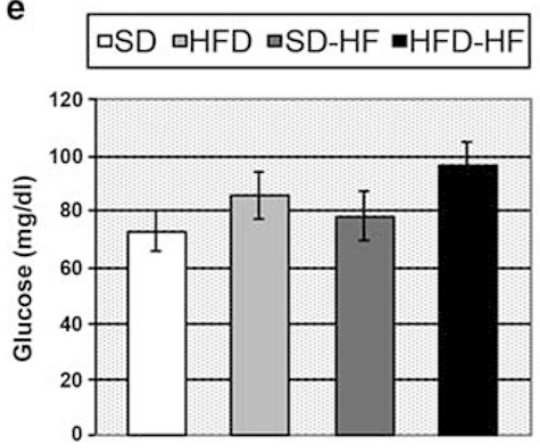

C

口SD 口HFD $\square$ SD-HF =HFD-HF

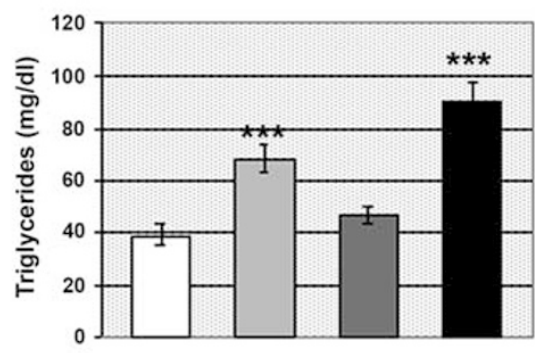

f
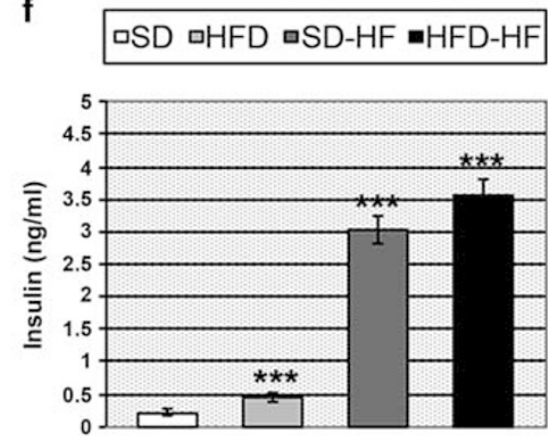

Figure 1 Body weight (a), and serum levels of ALT (b), triglycerides (c), total cholesterol (d), glucose (e) and insulin (f) in rats treated with different dietetic regimens. ${ }^{*}$-value $<0.05,{ }^{* * * P}$-value $<0.001$.
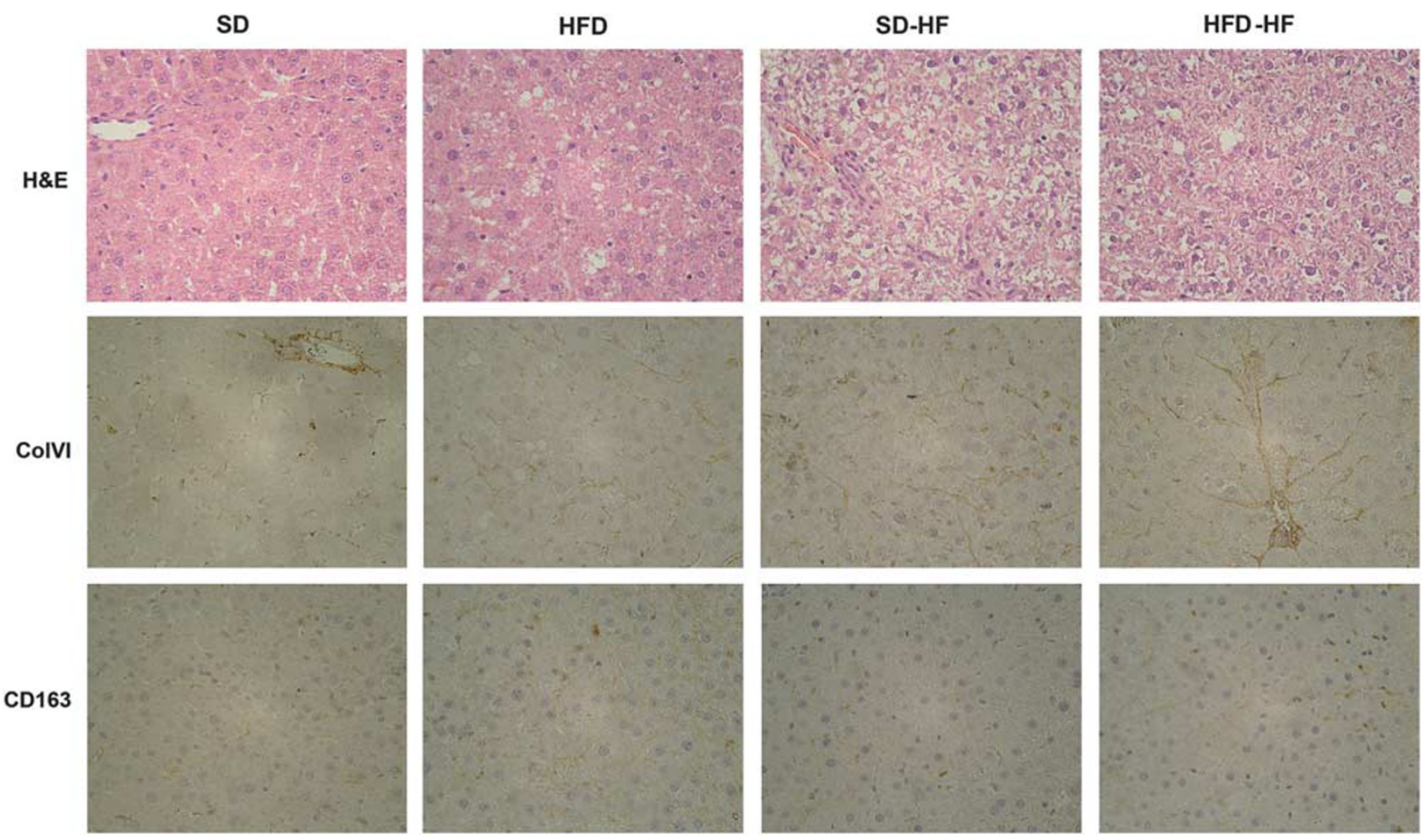

Figure $2 \mathrm{H} \& \mathrm{E}$ staining of liver tissue from rats treated with different dietetic regimens. Collagen Vl, and CD163 staining of liver tissue from rats treated with different dietetic regimens. SD, standard diet; HFD, high-fat diet; SD-HF, standard diet enriched with fructose; HFD-HF, high-fat diet enriched with high content of fructose. $\times 400$ Magnification. 

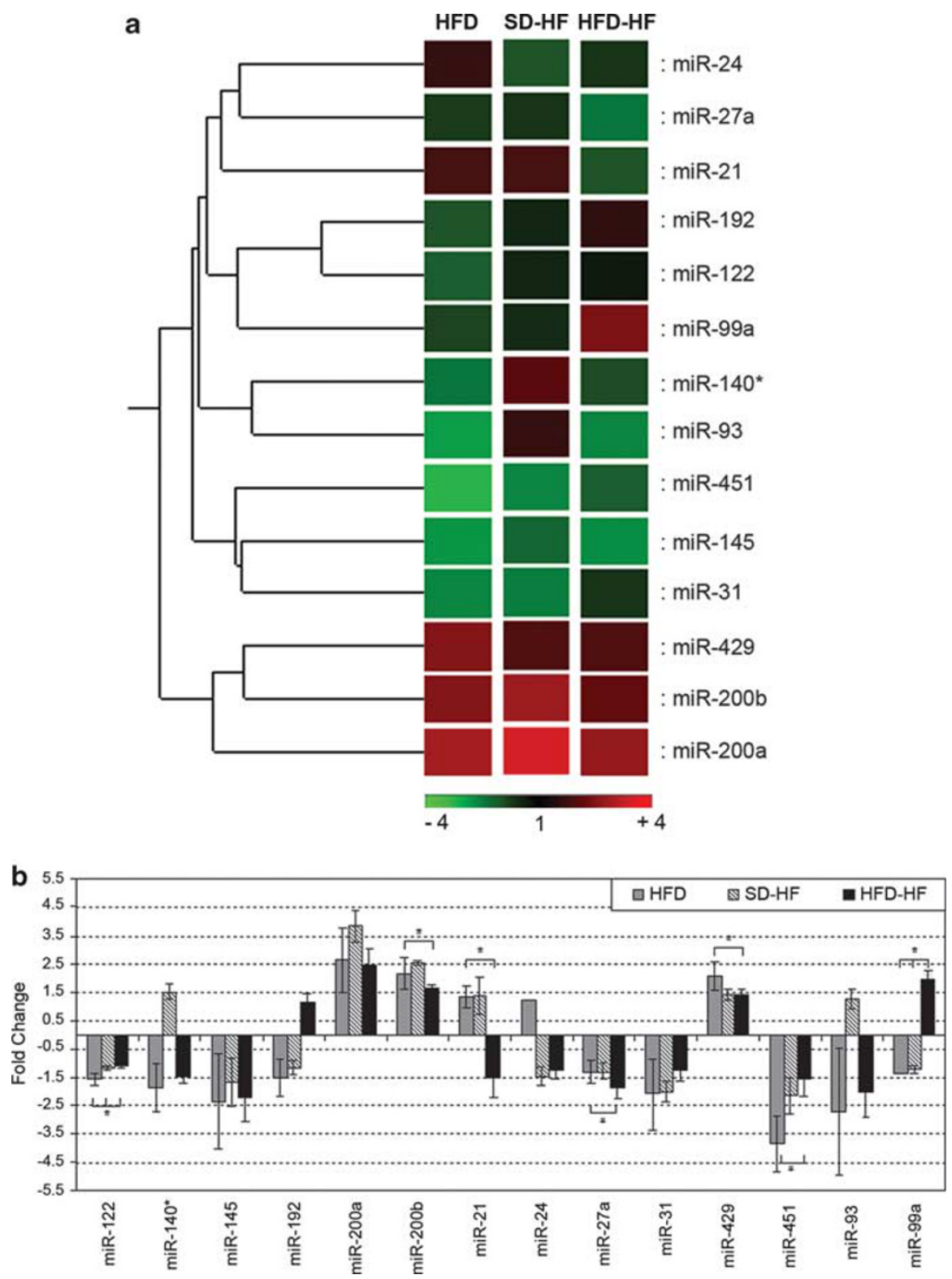

Figure 3 (a) Unsupervised hierarchical clustering of modulated miRNAs in different diets. Clustered miRNAs are listed on the right. The expression intensity of each miRNA varies from red to green: Red color means an expression value upregulated with respect to the control (SD) and green color means the opposite. (b) MiRNAs upregulated or downregulated in at least one treatment with a 1.4-fold. Fold change was calculated dividing the normalized signals of treated samples by the control sample. Asterisk $\left(^{*}\right)$ indicates $P$-values, calculated by ANOVA, $<0.05$ for miRNAs that are differently expressed in combined diet HFD-HF with respect to the single HFD or HF diet.

CD163 (marker of activated Kupffer cells). As reported in Figure 2, increased positivity for collagen VI and CD163 was observable only in liver tissues from rats treated with combined diet (HFD-HF).

\section{miRNA Expression Profiling and Functional Classification of Their Targets}

To identify possible variations in the expression pattern of miRNAs associated to diet-induced hepatic damage, we performed a large-scale expression profiling analysis of 350 rat mature miRNAs on liver tissue from SD, HFD, SD-HF and HDF-HF rats. We performed an unsupervised hierarchical clustering on miRNA expression data (Figure 3a). Fourteen miRNAs resulted significant $(P<0.05)$ and deregulated with a fold change of at least 1.4 in at least one treatment, with respect to the control. Fold change and statistical significance were reported in Figure $3 \mathrm{~b}$.

In particular, we found that miR-27a, miRNA-122, miR451, miR-145, miR-31, miR-429, miR-200a and miR-200b were similarly deregulated in all treatments, and other three miRNAs (miRNA-21, mR-192 and miR-99a) were significantly altered in HFD-HF combined diet $v s$ single diets 
(HFD or SD-HF). Other three miRNAs (miR-24, miR-140* and miR-93) were variably expressed. These miRNAs were used for investigating the association between their targets and pathways involved in the pathogenesis of NAFLD. For each miRNA, targets obtained by the bioinformatic prediction were classified according to biological processes correlated with the disease. Among biological processes mainly correlated to NAFLD we included apoptosis, calciummediated signaling, carbohydrate metabolism, cell glucose homeostasis, cell homeostasis, cell-cell signaling, cytokine and chemokine-mediated signaling pathway, defense response, fatty acid metabolism, intracellular signaling, lipid metabolism, regulation of cell cycle, response to stress and signal transduction (see Supplementary Table S2).

\section{Real-time Validation of miRNA Expression}

For the real-time PCR validation, we selected those miRNAs associated with at least three biological processes considered above (miR-122, miR-27a, miR-200a and miR-429) (Figure 4). We also included miR-200b and miR-451 because the former belongs to miR-200b-429 cluster, and the latter has the lowest expression in all dietetic regimens with respect to the control. Finally, we included in the real-time PCR validation also miR21, miR-99a and miR-192, significantly altered upon combined diet (HFD-HF) with respect to single diets.

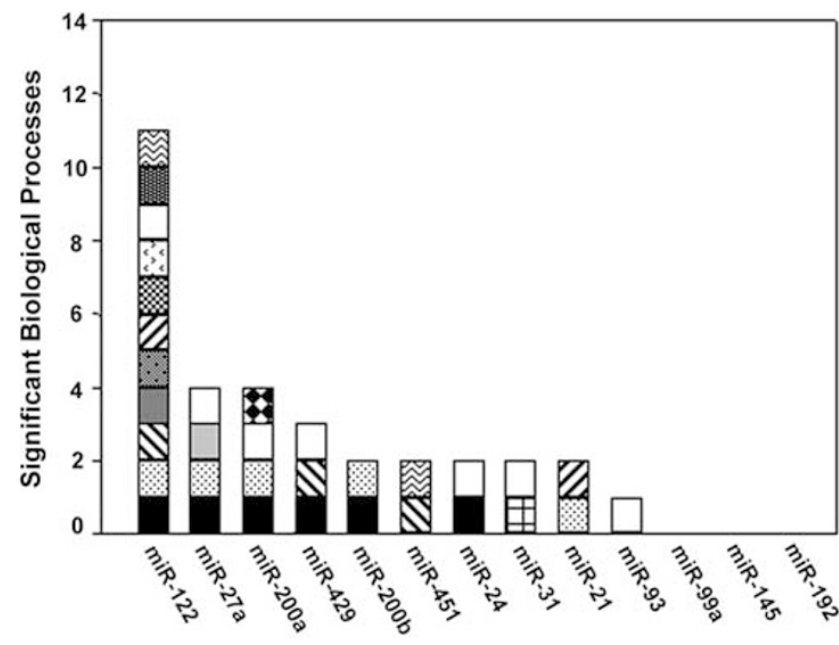

Legend

apoptosis (GO:0006915)
calcium mediated signaling (GO:0019722)
carbohydrate metabolism (GO:0005975)
cell glucose homeostasis (GO:0001678)
cell homeostasis (GO:0019725)
cell-cell signaling (GO:0007267)
cytokine and chemokine mediated signaling pathway (GO:0019221)
defense response (GO:0006952)
fatty acid metabolism (GO:0006631)
intracellular signaling cascade (GO:0007242)
lipid metabolism (GO:0006629)
regulation of cell cycle (GO:0000074)
response to stress (GO:0006950)
signal transduction (GO:0007165)

Figure 4 Significant upregulated or downregulated miRNAs associated with biological processes relevant in NAFLD pathogenesis.
Real-time PCR confirmed that miR-122, miR-451 and miR-27a were downregulated in HFD, SD-HF and HFD-HF with respect to the SD (Figure $5 \mathrm{a}-\mathrm{c}$ ), whereas miR-429, miR200a and miR-200b were upregulated in all hypercaloric regimens compared with the control (SD) (Figure 5d-f). Interestingly, miR-21 was downregulated only in diets enriched with fructose (Figure $5 \mathrm{~g}$ ). Moreover, the differential expression of these miRNAs in all hypercaloric regimens was significant $(P<0.05)$ compared with SD. No statistically significant variations in the expression of miR-99a and miR192 were found (Figure $5 \mathrm{~h}$ and i).

\section{Relationship Between miRNAs and Proteins Potentially Determinant in NAFLD}

It has been already reported that microarray experiments might reveal the systematic inverse correlation between the miRNA expression and their potential target transcripts. ${ }^{35-37}$ However, as shown in specific public databases (miRBase and TarBASE), each miRNA has been predicted or validated for the regulation of several molecular targets. Thus, the validation of all 'real' miRNA targets in a specific in vivo system should require very demanding and expensive analyses. Here, we found a large number of rat-specific targets for miRNA-122, miRNA-200a, miRNA-200b, miRNA-429, miRNA-451, miRNA-27a from bioinformatic prediction. Thus, to bypass the complication because of the surplus of possible targets we refined data, and then we focused the analysis only on some molecules with a potential determinant biological role in diet-induced NAFLD. In Figure 6, we graphicated the relationship between a specific miRNA, molecular targets and biological processes for a few interesting molecules potentially involved in NAFLD development. Interestingly, some of these determinants, such as lipopolysaccharide-induced tumor necrosis factor $\alpha$ (LITAF) and phosphatase and tensin homolog (PTEN), might be common targets for multiple miRNAs (cooperativity) and might be involved in different metabolic processes (ie lipid metabolism, apoptosis and cytokine/chemokine-mediated signaling pathway).

Among various targets, we validated protein kinase C (PKC) $\delta$, peroxisome proliferator-activated receptor (PPAR) $\gamma$, LITAF, ALDOLASE-A, p38 mitogen-activated protein kinase (p38MAPK), PTEN, LIPIN1, EFNA1 (EPHRIN-A1), EPHA2, FLT-1 (also named vascular endothelial growth factor receptor-1), RICTOR, TP53, STAT1 and STAT2 by western blotting analysis, performed on liver tissue from rats treated with different dietetic regimens. The expression levels of RICTOR, TP53, STAT1, STAT2 and PPAR $\gamma$ were similar in SD and in all hypercaloric diets (HFD, SD-HF and HFD-HF) (see Supplementary Figure S1). On the other hand, the analysis of protein expression pattern of $\mathrm{PKC} \delta$, LITAF, ALDOLASE-A, PTEN, LIPIN1, EPHRIN-A1 and EPHA2 revealed, as expected, an anti-parallel trend with respect to their associated miRNAs (Figure 7). In particular, an increased expression of PKC $\delta$, LITAF, ALDOLASE-A and 


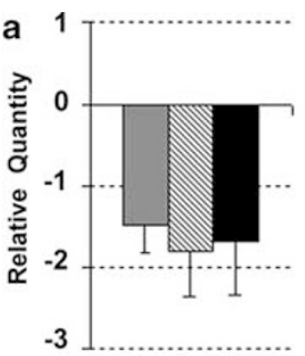

miR-122

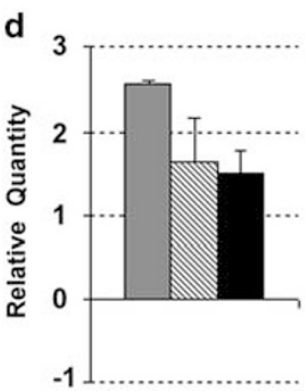

$\operatorname{miR}-429$

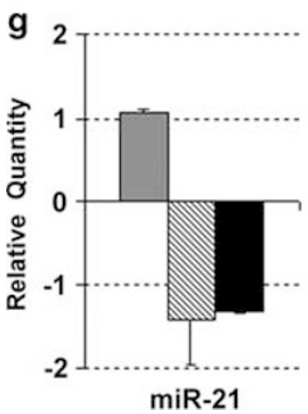

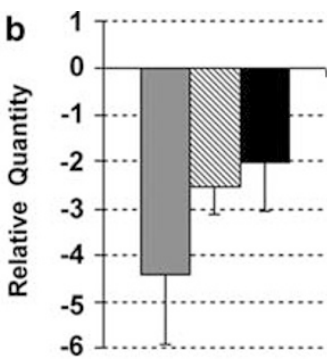

miR-451

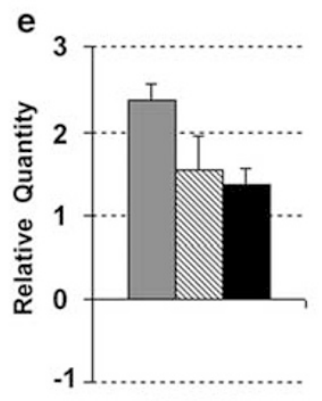

miR-200a

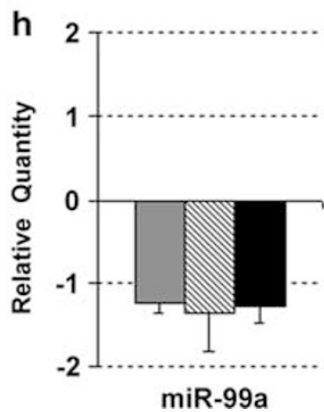

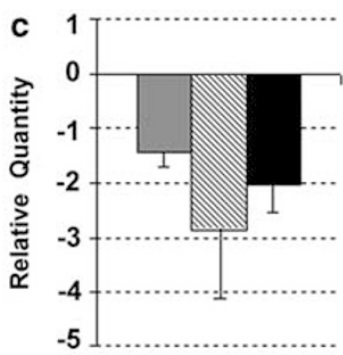

miR-27a

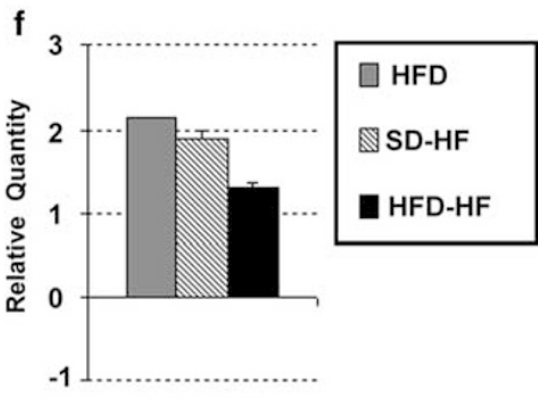

miR-200b

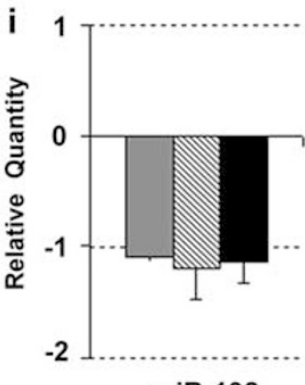

miR-192

Figure 5 Real-time PCR validation of upregulated and downregulated miRNAs. Relative quantity of all miRNAs (a-i) is reported with respect to the control considered as 0. MiRNAs of HFD, SD-HF and HFD-HF, reported in panels (a-g), are differentially expressed with significant $P$ ( $<0.05$ ) vs control.

p38MAPK was found in all HFD, SD-HF and HFD-HF rats, whereas protein levels of PTEN, LIPIN1, FLT-1, EPHRIN-A1 and EPHA2 are greatly downregulated in hypercaloric regimens with respect to the control diet (Figure 7).

\section{Assessment of mRNAs Encoding for Novel Determinants in NAFLD}

To investigate whether the effect on protein expression, following the different diets, is attributable to variations of miRNAs, mRNAs or both, we assessed, by real-time PCR, the expression levels of nine mRNAs associated to investigated proteins (Figure 8). Only LITAF, PTEN, LIPIN1, EPHRINA1, EPHA2 and FLT1 mRNA showed a statistically significant deregulation $(P<0.05)$ greater than \pm 1.5 fold of change in at least one of dietetic regimen.

\section{DISCUSSION}

NAFLD is a multifactorial disease predominantly regulated by the interplay of genetic predisposition and environmental factors. $^{38}$ It is commonly accepted that the molecular mechanisms involved in the development and progression of NAFLD are similar to those leading to the obesity and metabolic syndrome. In fact, the dysregulation of lipid metabolism, insulin signaling, inflammatory response and immune response have important role in the onset and outcome of NAFLD. ${ }^{7,8}$ The era of high-throughput technologies has revolutionized the common approach to studying NAFLD pathogenesis. In particular, miRNA expression patterns have been associated to different stages of NAFLD. ${ }^{24,25}$

Here, we identified some differentially expressed miRNAs in diet-induced model of NAFLD. We found that rats fed HFD or SD-HF showed, respectively, only a mild steatosis or ballooning. In HFD-HF rats, the state was aggravated by the sum of these histopathological conditions; but also by the presence of perisinusoidal fibrosis as indicated by the increased collagen VI component, and by the increase of CD163-positive cells in liver parenchyma suggestive of 


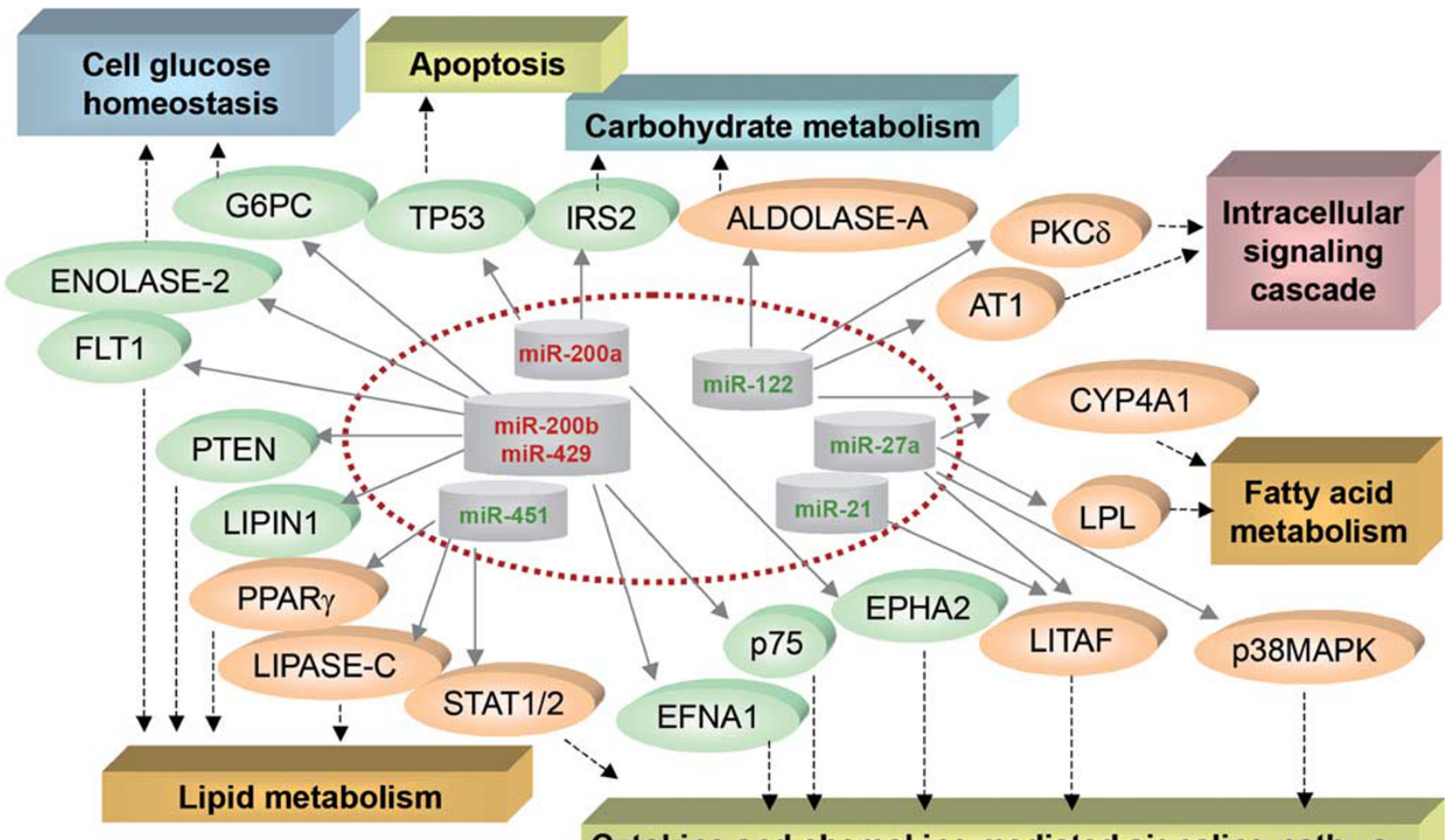

Cytokine and chemokine mediated signaling pathway

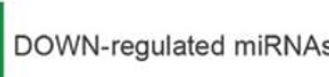

UP-regulated miRNAs

UP-regulated targets

DOWN-regulated targets

Figure 6 Schematic representation of potential determinants in the pathogenesis of NAFLD. Predicted targets were obtained from different prediction programs including TargetScan, miRanda and miRecords. All molecular targets were functionally classified using gene ontology annotation.

a pre-inflammatory state. The levels of miRNAs potentially regulating the transcripts of factors involved in fatty acid metabolism, inflammatory cytokines, cell growth and apoptosis, signal transduction and fibrogenesis were significantly altered by all hypercaloric regimens, especially in HFD-HF rats. In keeping with a previous published study, we confirmed the downregulation of miR-122. ${ }^{26}$ In liver, miR-122 regulates several targets affecting lipid homeostasis, including fatty acid synthase, 3-hydroxy-3-methyl-glutarylcoenzyme A reductase and their transcriptional activators, the sterolresponse-element binding protein-1c and 2. Therefore, miR-122 downregulation may have a significant role in the pathogenesis of the disease. However, downregulation of miR-122 is not a distinctive signature of NASH; in fact, has been previously described in hepatocellular carcinoma, suggesting its potential role in liver cancer development. ${ }^{39} \mathrm{In}$ addition, it has been recently reported that the hepatic transcription of miR-122 locus is under circadian control. ${ }^{40}$ The increased expression of levels of miR-200a, miR-200b, miR-429, belonging to the same genomic cluster, were found in our model of NAFLD, and it is in agreement with other studies. $^{26,27}$ Contrarily from that observed by Vinciguerra et $a l,{ }^{41}$ but equally to Li et $a l^{26}$ we observed a significant downregulation of miR-21 that seems to be unrelated to PTEN expression, but to other potential targets such as LITAF. Recently, an interesting role in liver fibrogenesis was attributed to the upregulation of miR-27a and miR-27b ${ }^{42}$; in our model, the downregulation of miR-27a appears mainly associated to the inflammatory molecules and fatty acid metabolism. The role of the downregulated miR-451 observed in diet-induced NAFLD, as well as in human $\mathrm{NASH},{ }^{27}$ still remains obscure. In fact, in our study we have not identified any potential-associated proteins/mRNA.

Herein, we demonstrated deregulation of other interesting miRNAs in NAFLD. On the basis of our bioinformatic target prediction, these miRNAs may have a role in the regulation of biological processes closely related to NAFLD pathogenesis. Noteworthy, we found that predicted targets, such as $\operatorname{PPAR} \gamma, \operatorname{PKC} \delta$, ALDOLASE-A and LITAF were upregulated in HFD, SD-HF and HFD-HF rats. Protein levels of PTEN, LIPIN1, EPHA2 and EPHRIN-A were instead decreased in rats treated with hypercaloric regimens. PPAR $\gamma, \operatorname{PTEN}$ and $\mathrm{PKC} \delta$ are well known molecular actors in NAFLD. PPAR $\gamma$ belongs to a family of transcription factors involved in the regulation of intracellular fatty acid oxidation and storage. ${ }^{43}$ In agreement with our miRNA data, the hepatic expression 
a

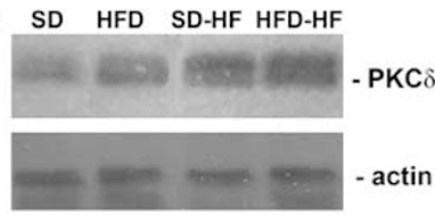

b

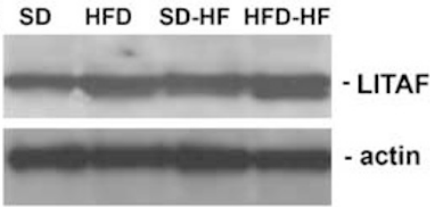

C SD HFD SD-HF HFD-HF

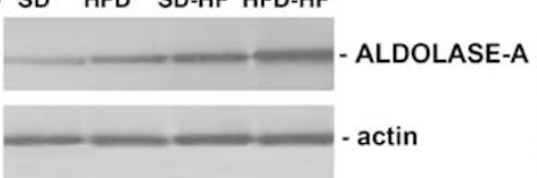

d SD HFD SD-HF HFD-HF

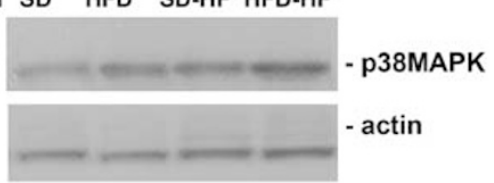

e

SD HFD SD-HF HFD-HF

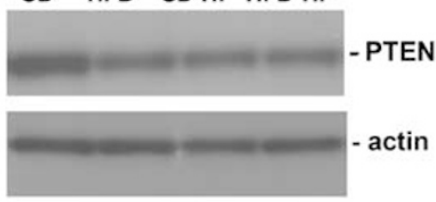

f SD HFD SD-HF HFD-HF

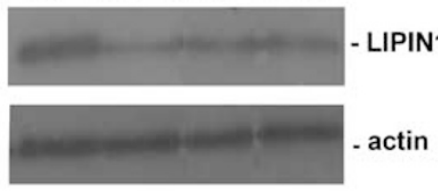

g

SD HFD SD-HF HFD-HF
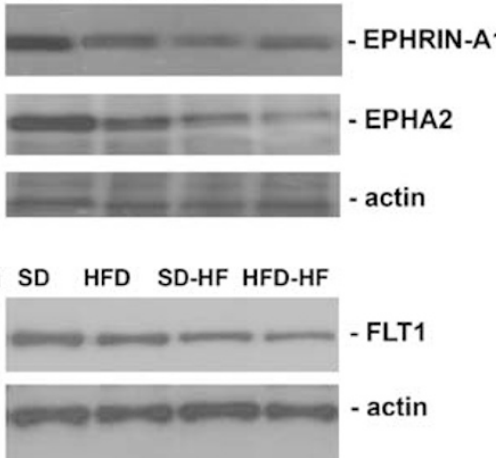

h SD HFD SD-HF HFD-HF
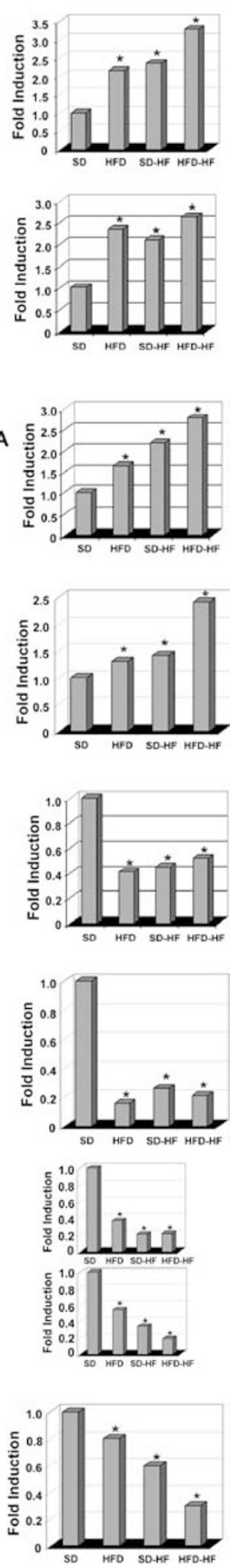

Figure 7 Representative western blot for PKC $\delta$ (a), LITAF (b), ALDOLASE-A (c), p38MAPK (d), PTEN (e), LIPIN1 (f), EPHRIN-A1 and EPHA2 (g) and FLT1 (h) in rats treated with SD, HFD, SD-HF and HFD-HF. $\beta$-Actin profiles were reported as loading control. The left panels show the representative profiles of at least five independent experiments, and the right panels show the densitometric analysis performed in quadruplicate.

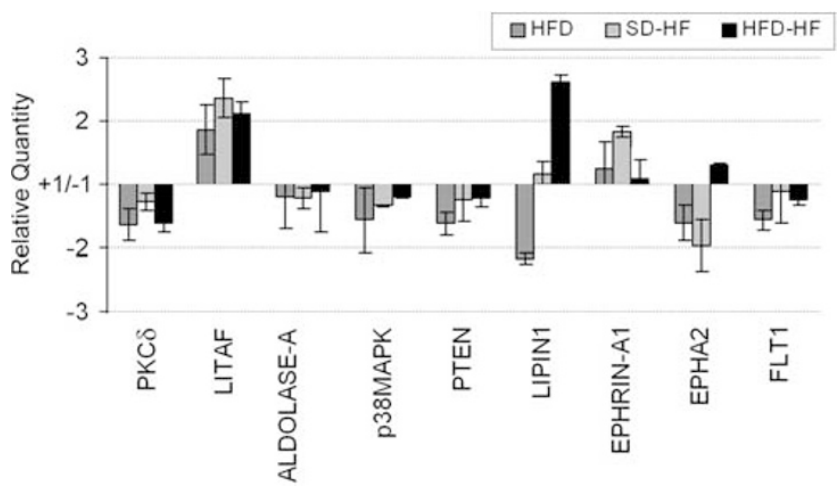

Figure 8 Real-time PCR validation of upregulated and downregulated mRNAs. The histogram reports the relative quantity of all mRNAs with respect to the control, considered as 0 . Values between +1 and -1 indicating invariant expression are not shown. Data are statistically significant $(P<0.05)$ for relative quantity greater than \pm 1.5 in at least one of the dietetic regimen.

levels of PPAR $\gamma$ mRNA and protein were upregulated by high-fat diet. ${ }^{44}$ PTEN is a phosphatase that antagonizes the PI3K/AKT signaling pathway by dephosphorylating phosphatidylinositol-3,4,5-triphosphate. PTEN is downregulated in models of NAFLD and its knockdown predispose mice to develop NASH and hepatocellular carcinoma. ${ }^{45,46}$ Finally, it is not surprising to find the hepatic $\mathrm{PKC} \delta$ upregulation in diet-induced NAFLD, because this protein is actively involved in the regulation of hepatic insulin resistance. ${ }^{47}$ However, in addition to these already known proteins, our study revealed the deregulation of other new molecular determinants that could be involved in the development of NAFLD, as well as in its progression to advanced fibrosis and hepatocellular carcinoma. For example, LIPIN1 deficiency results in defective adipogenesis in cases of mouse fatty liver dystrophy and human lipodystrophy, whereas polymorphisms in its encoding gene was found associated to metabolic syndrome. ${ }^{48,49}$ Thus, defects in LIPIN1 gene could be signs of an individual predisposition to make NAFLD in the presence of an excessive dietary intake. The data on LITAF and its possible upregulation during diet-induced NAFLD is also very interesting. LITAF, in fact, has been identified as a transcription factor activated by lipopolysaccharide and capable of activating the promoter of several proinflammatory cytokines. ${ }^{50}$ In our system, especially in conditions characterized by excessive dietary intake of fructose, LITAF might be one of the key players that links an increased endotoxemia, toll-like receptor-4, necro-inflammation and the development of NASH. ${ }^{51}$ Interestingly, the increased expression of ALDOLASE-A could be correlated in part with the activation of hypoxia-inducible factor-1 (HIF-1) $\alpha$. Indeed, HIF- $1 \alpha$, considered as an important regulator of profibrotic and vasoactive mediators in hypoxic hepatocytes, mediates the increased expression of ALDOLASE-A in endotoxemic rats. $^{52,53}$ On the other hand, ALDOLASE-A, usually absent in the adult liver, might be an early signal that 
precedes neoplastic transformation and development of hepatocellular carcinoma, which occurs in some very advanced stages of NASH. ${ }^{54}$ Worthy of further studies is the hepatic expression level of EPHRIN-A1 and its receptor during dietinduced NAFLD, because of the many cellular functions that these proteins pursue in physiological and pathological conditions. ${ }^{55,56}$ The real role of p38MAPK upregulation in the NAFLD pathogenesis is still unclear even though it was previously demonstrated that this kinase together with ERK is remarkably activated in rodent models of NAFLD. ${ }^{57}$ Our data, agree with other studies which found that hepatic expression of FLT1 mRNA was decreased in obese mice compared with lean mice and suggest a potential involvement of vascular endothelial growth factor and its receptor in the pathogenesis of NAFLD. ${ }^{58}$

In a general view, it is recognized that protein expression is directly correlated to the expression of its cognate mRNA, which in turn generally is inversely correlated to the expression of its regulatory miRNA(s). Our data demonstrated that this phenomenon is plausible to explain LITAF increase, PTEN and FLT1 decrease, in all hypercaloric regimens. In the case of LIPIN1 and EPHA2, the antiparallel trend between miRNA and mRNA/protein is straight only in some dietetic regimens. On the other hand, although the protein expression of EPHRIN-A1 seems to be inverse to miR-200b-429 cluster, the mRNA expression is uncorrelated either to these miRNAs, or protein levels. In all these cases, the apparent discrepancy between miRNAs and mRNA/protein expression might be explained with the involvement of miRNAs acting as post-transcriptional repressors. ${ }^{59,60}$

Our findings demonstrate that some miRNAs and some of their targets may correlate with the development of fatty liver and/or progression of nutritional steatohepatitis. Obviously, further investigations will be required to completely understand the association among miRNA profiles, protein targets and NAFLD pathogenesis. These should include (1) validation of protein expression changes in individuals with different stages of diseases and (2) in vitro experiments for miRNA-mRNA interaction assessment.

Furthermore, our results highlight the importance of experimental/bioinformatics to decipher the myriad of data and to solve both physiologically and pathologically relevant issues. ${ }^{61}$ In our case, the information of mirnome analysis represents a good starting point to better understand the pathogenetic mechanisms of NAFLD, as well as to identify potential therapeutic targets, early diagnostic markers of NASH, and finally predictors of hepatocellular carcinoma.

Supplementary Information accompanies the paper on the Laboratory Investigation website (http://www.laboratoryinvestigation.org)

\section{ACKNOWLEDGEMENT}

This work was entirely supported by Bambino Gesù Children's Hospital and Research Institute, Rome, Italy.

\section{DISCLOSURE/CONFLICT OF INTEREST}

The authors declare no conflict of interest.

1. Argo CK, Caldwell SH. Epidemiology and natural history of nonalcoholic steatohepatitis. Clin Liver Dis 2009;13:511-531.

2. Brunt EM. Nonalcoholic steatohepatitis: definition and pathology. Semin Liver Dis 2001;21:3-16.

3. Bondini S, Kleiner DE, Goodman ZD, et al. Pathologic assessment of non-alcoholic fatty liver disease. Clin Liver Dis 2007;11:17-23, vii.

4. Paradis V, Bedossa P. Definition and natural history of metabolic steatosis: histology and cellular aspects. Diabetes Metab 2008;34: 638-642.

5. Utzschneider KM, Kahn SE. Review: the role of insulin resistance nonalcoholic fatty liver disease. J Clin Endocrin Metab 2006;91:4753-4761.

6. Erickson SK. Nonalcoholic fatty liver disease. J Lipid Res 2009;50: S412-S416.

7. Malaguarnera M, Di Rosa M, Nicoletti F, et al. Molecular mechanisms involved in NAFLD progression. J Mol Med 2009;87:679-695.

8. Cheung O, Sanyal AJ. Recent advances in nonalcoholic fatty liver disease. Curr Opin Gastroenterol 2009;25:230-237.

9. Alisi A, Marcellini M, Nobili V. Bioinformatics as tool to identify gene/ protein-pathways associated with nonalcoholic fatty liver disease/ nonalcoholic steatohepatitis. Hepatology 2007;46:1306.

10. Baranova A, Schlauch $K$, Elariny $H$, et al. Gene expression patterns in hepatic tissue and visceral adipose tissue of patients with nonalcoholic fatty liver disease. Obes Surg 2007;17:1111-1118.

11. Baranova A, Liotta L, Petricoin E, et al. The role of genomics and proteomics: technologies in studying non-alcoholic fatty liver disease. Clin Liver Dis 2007;11:209-220.

12. Barbato $C$, Arisi I, Frizzo ME, et al. Computational challenges in miRNA target predictions: to be or not to be a true target? J Biomed Biotechnol 2009;2009:803069.

13. Calvert VS, Collantes R, Elariny $\mathrm{H}$, et al. A systems biology approach to the pathogenesis of obesity-related nonalcoholic fatty liver disease using reverse phase protein microarrays for multiplexed cell signaling analysis. Hepatology 2007;6:166-172.

14. Alisi A, Masotti A, Nobili V. Profiling microRNA expression: a snapshot of nonalcoholic steatohepatitis and a recording of its pathogenesis. Hepatology 2009;49:706-707.

15. Lee RC, Feinbaum RL, Ambros V. The C. elegans heterochronic gene lin-4 encodes small RNAs with antisense complementarity to lin-14. Cell 1993;75:843-854.

16. Ambros V. The functions of animal microRNAs. Nature 2004;431: 350-355.

17. Bartel DP. MicroRNAs: genomics, biogenesis, mechanism, and function. Cell 2004;116:281-297.

18. Chang TC, Mendell JT. microRNAs in vertebrate physiology and human disease. Annu Rev Genomics Hum Genet 2007;8:215-239.

19. Nelson KM, Weiss GJ. MicroRNAs and cancer: past, present, and potential future. Mol Cancer Ther 2008;7:3655-3660.

20. $\mathrm{Xu}$ P, Guo M, Hay BA. MicroRNAs and the regulation of cell death. Trends Genet 2004;20:617-624.

21. Karp X, Ambros V. Encountering microRNAs in cell fate signaling. Science 2005;310:1288-1289.

22. Iorio MV, Croce CM. MicroRNAs in cancer: small molecules with a huge impact. J Clin Oncol 2009;27:5848-5856.

23. Pandey AK, Agarwal $P$, Kaur K, et al. MicroRNAs in diabetes: tiny players in big disease. Cell Physiol Biochem 2009;23:221-232.

24. Bala S, Marcos M, Szabo G. Emerging role of microRNAs in liver diseases. World J Gastroenterol 2009;15:5633-5640.

25. Chen XM. MicroRNA signatures in liver diseases. World J Gastroenterol 2009;15:1665-1672.

26. Li S, Chen X, Zhang H, et al. Differential expression of microRNAs in mouse liver under aberrant energy metabolic status. J Lipid Res 2009;50:1756-1765.

27. Cheung O, Puri P, Eicken C, et al. Nonalcoholic steatohepatitis is associated with altered hepatic microRNA expression. Hepatology 2008;48:1810-1820.

28. Jin X, Ye Y-F, Chen S-H, et al. MicroRNA expression pattern in different stages of nonalcoholic fatty liver disease. Dig Liver Dis 2009;41: 289-297. 
29. Pogribny IP, Starlard-Davenport A, Tryndyak VP, et al. Difference in expression of hepatic microRNAs miR-29c, miR-34a, miR-155, and miR$200 \mathrm{~b}$ is associated with strain-specific susceptibility to dietary nonalcoholic steatohepatitis in mice. Lab Invest 2010. Epub ahead of print.

30. Brunt EM. Grading and staging the histopathological lesions of chronic hepatitis: the Knodell histology activity index and beyond. Hepatology 2000;31:241-246.

31. Bolstad BM, Irizarry RA, Astrandand MT, et al. A comparison of normalization methods for high density oligonucleotide array data based on variance and bias. Bioinformatics 2003;19:185-193.

32. Livak KJ, Schmittgen TD. Analysis of relative gene expression data using real-time quantitative PCR and the 2(-Delta Delta $C(T)$ ) method. Methods 2001;25:402-408.

33. Thomas PD, Campbell MJ, Kejariwal A, et al. PANTHER: a library of protein families and subfamilies indexed by function. Genome Res 2003:13:2129V2141.

34. Alisi A, Spagnuolo S, Leoni S. Treatment with EGF increases the length of S-Phase after partial hepatectomy in rat, changing the activities of cdks. Cell Physiol Biochem 2003;13:239-248.

35. Lim LP, Lau NC, Garrett-Engele P, et al. Microarray analysis shows that some microRNAs downregulate large numbers of target mRNAs. Nature 2005;433:769-773.

36. Wang X. Systematic identification of microRNA functions by combining target prediction and expression profiling. Nucleic Acids Res 2006;34:1646-1652.

37. Sood P, Krek A, Zavolan $M$, et al. Cell-type-specific signatures of microRNAs on target mRNA expression. Proc Natl Acad Sci USA 2006;103:2746-2751.

38. Wilfred de Alwis NM, Day CP. Genetics of alcoholic liver disease and nonalcoholic fatty liver disease. Semin Liver Dis 2007;27:44-54.

39. Kutay H, Bai S, Datta J, et al. Downregulation of miR-122 in the rodent and human hepatocellular carcinomas. J Cell Biochem 2006;99:671-678.

40. Gatfield D, Le Martelot $G$, Vejnar $C E$, et al. Integration of microRNA miR-122 in hepatic circadian gene expression. Genes Dev 2009;23: 1313-1326.

41. Vinciguerra M, Sgroi A, Veyrat-Durebex C, et al. Unsaturated fatty acids inhibit the expression of tumor suppressor phosphatase and tensin homolog (PTEN) via microRNA-21 up-regulation in hepatocytes. Hepatology 2009;49:1176-1184.

42. Ji J, Zhang J, Huang G, et al. Over-expressed microRNA-27a and 27b influence fat accumulation and cell proliferation during rat hepatic stellate cell activation. FEBS Lett 2009;583:759-766.

43. Lee $\mathrm{CH}$, Olson P, Evans RM. Minireview: lipid metabolism, metabolic diseases, and peroxisome proliferator-activated receptors. Endocrinology 2003;144:2201-2207.

44. Yu S, Matsusue K, Kashireddy P, et al. Adipocyte-specific gene expression and adipogenic steatosis in the mouse liver due to peroxisome proliferator-activated receptor gamma1 (PPARgamma1) overexpression. J Biol Chem 2003;278:49-505.
45. Vinciguerra M, Foti M. PTEN at the crossroad of metabolic diseases and cancer in the liver. Ann Hepatol 2008;7:192-199.

46. Watanabe $S$, Horie $Y$, Kataoka $E$, et al. Non-alcoholic steatohepatitis and hepatocellular carcinoma: lessons from hepatocyte-specific phosphatase and tensin homolog (PTEN)-deficient mice. J Gastroenterol Hepatol 2007;22:S96-S100.

47. Meshkani R, Adeli K. Hepatic insulin resistance, metabolic syndrome and cardiovascular disease. Clin Biochem 2009:42:1331-1346.

48. Peterfy M, Phan J, Xu P, et al. Lipodystrophy in the fld mouse results from mutation of a new gene encoding a nuclear protein, lipin. Nat Genet 2001;27:121-124.

49. Wiedmann $S$, Fischer $M$, Koehler $M$, et al. Genetic variants within the LPIN1 gene, encoding lipin, are influencing phenotypes of the metabolic syndrome in humans. Diabetes 2008:57:209-217.

50. Tang X, Marciano DL, Leeman SE, et al. LPS induces the interaction of a transcription factor, LPS-induced TNF-alpha factor, and STAT6(B) with effects on multiple cytokines. Proc Natl Acad Sci USA 2005;102: 5132-5137.

51. Alisi A, Panera N, Nobili V. Toll-like receptor 4: a starting point for proinflammatory signals in fatty liver disease. Hepatology 2010;51:714-715.

52. Copple BL, Bustamante JJ, Welch TP, et al. Hypoxia-inducible factordependent production of profibrotic mediators by hypoxic hepatocytes. Liver Int 2009;29:1010-1021.

53. Scharte M, Han X, Uchiyama T, et al. LPS increases hepatic HIF-1alpha protein and expression of the HIF-1-dependent gene aldolase $\mathrm{A}$ in rats. J Surg Res 2006;135:262-267.

54. Castaldo G, Calcagno G, Sibillo R, et al. Quantitative analysis of aldolase A mRNA in liver discriminates between hepatocellular carcinoma and cirrhosis. Clin Chem 2000:46:901-906.

55. Ivanov Al, Romanovsky AA. Putative dual role of ephrin-Eph receptor interactions in inflammation. IUBMB Life 2006:58:389-394.

56. Wykosky J, Debinski W. The EphA2 receptor and ephrinA1 ligand in solid tumors: function and therapeutic targeting. Mol Cancer Res 2008;6:1795-1806.

57. Kushiyama A, Shojima N, Ogihara T, et al. Resistin-like molecule beta activates MAPKs, suppresses insulin signaling in hepatocytes, and induces diabetes, hyperlipidemia, and fatty liver in transgenic mice on a high fat diet. J Biol Chem 2005;280:42016-42025.

58. Redaelli CA, Semela D, Carrick FE, et al. Effect of vascular endothelial growth factor on functional recovery after hepatectomy in lean and obese mice. J Hepatol 2004;40:305-312.

59. Filipowicz W, Bhattacharyya SN, Sonenberg N. Mechanisms of posttranscriptional regulation by miRNAs: are the answers in sight? Nat Rev Genet 2008;9:102-114.

60. Pillai RS. MiRNA function: multiple mechanisms for a tiny RNA? RNA 2005;11:1753-1761.

61. Masotti A, Da Sacco L, Bottazzo GF, et al. Microarray technology: a promising tool in the field of nutrigenomics discoveries. Crit Rev Food Sci Nutr 2010;50:693-698. 